\title{
Kombinierte radiologische und nuklearmedizinische Bildgebung in Tierexperimenten: Ein Überblick über die aktuellen Möglichkeiten
}

\author{
Combination of Radiological And Nuclear Medical Imaging in Animals: \\ An Overview About the Today's Possibilties
}

Autoren

Institute
M. Behe' ${ }^{1}$, B. Keil' , H. Alfke ${ }^{3}$, I. Böhm ${ }^{4}$, A. Kiessling², M. Gotthardt ${ }^{5}$, J. T. Heverhagen ${ }^{2}$

Klinik für Nuklearmedizin, Philipps-Universität Marburg

Klinik für Strahlendiagnostik, Philipps-Universität Marburg

Klinikum Lüdenscheid, Klinik für Diagnostische und Interventionelle Radiologie

${ }^{4}$ AG Molecular Imaging, Radiologische Universitätsklinik Bonn

Department of Nuclear Medicine, Radboud University Nijmegen
Key words

- MR imaging

- radionuclide imaging

- SPECT

- animal investigations

- CT

- PET CT eingereicht 20.2.2007

akzeptiert 3.5.2007

Bibliografie

DOI $10.1055 / \mathrm{s}-2007-963263$

Online-Publikation: 2007

Fortschr Röntgenstr 2007; 179:

796-803 @ Georg Thieme

Verlag KG Stuttgart · New York ·

ISSN 1438-9029

Korrespondenzadresse

Dr. Martin Behe

Klinik für Nuklearmedizin,

Philipps-Universität Marburg

Baldingerstraße

35043 Marburg

Tel.: ++49/6421/2862808

Fax: $++49 / 6421 / 2862899$

behe@staff.uni-marburg.de

\section{Zusammenfassung}

$\nabla$

Die molekulare Bildgebung von Kleintieren hat in den letzten Jahren eine schnelle Entwicklung durchlaufen. Ein Grund dafür ist, dass verschiedene Forschungsgebiete davon profitieren können. Neben forschungstechnischen Gründen spielt dabei auch die Reduzierung der Tierzahl aus ethischen und finanziellen Gründen eine Rolle. Durch die nichtinvasiven Bildgebungsmethoden ist es möglich, ein Tier in einer Versuchsreihe mehrmals zu untersuchen, ohne dass das Tier getötet werden muss. Dadurch ist es möglich, im selben Tier die Entwicklung eines pathologischen Prozesses zu verfolgen. Die dabei angewendeten radiologischen Methoden, wie die Magnetresonanztomografie oder Computertomografie, aber auch die nuklearmedizinischen Methoden, wie die „Single Photon Emissions Computer Tomography“ oder „Positronen Emissions Tomography,“ weisen Nachteile auf. Bei den radiologischen Methoden ist die molekulare Aussage begrenzt, während die nuklearmedizinischen Methoden darunter leiden, dass es schwierig ist einen erhöhten Uptake einer anatomischen Lokalisation zuzuordnen. Das führt dazu, dass die Fusion der Methoden in vielen Fällen zu einem zusätzlichen Gewinn an Informationen führt. In dieser Übersichtsarbeit sollen die heutigen Möglichkeiten kombinierter Bildgebung und deren Vorteile aber auch die Nachteile aufgezeigt werden.

\section{Einleitung}

Die biomedizinische Bildgebung von Tiermodellen hat in der letzten Dekade deutlich an Bedeutung gewonnen. Im gleichen Maß hat sich auch der Einsatz von bildgebenden Methoden in der Forschung an Tiermodellen gesteigert. Die Entwicklungen auf diesem Gebiet versprechen, dass dieser Trend auch über die nächsten Jahre anhält.

\section{Abstract \\ $\nabla$}

Molecular imaging of small animals has made considerable progress in the last years. Various research fields are interested in imaging small animals due to the lower numbers of animals per experiment. This has advantages with respect to financial, ethical and research aspects. Non-invasive imaging allows examination of one animal several times during the same experiment. This makes it possible to follow a pathological process in the same animal over time. However, the radiological methods used such as magnetic resonance imaging or computed tomography as well as the nuclear medicine methods such as single photon emission computed tomography or positron emission tomography suffer from disadvantages. Molecular aspects are limited in the radiological methods while anatomical localization is difficult in nuclear medicine. The fusion of these methods leads to additional information. This review shows today's possibilities with their advantages as well as disadvantages.
Die Bildgebung erlaubt den Forschern dabei einen aktuellen Einblick in die Struktur und Funktionen von Organsystemen in vivo.

Die wichtigste Eigenschaft, welche die Bildgebung unersetzlich macht, ist die Tatsache, dass die Untersuchungen non-invasiv und damit uneingeschränkt wiederholbar sind. Dadurch sind Verlaufsstudien in den gleichen Tieren möglich. Dies führt zu einer signifikanten Reduktion der 
Zahl der benötigten Tiere um eine bestimmte Fragestellung zu beantworten. Darüber hinaus können unerwünschte Variationen dadurch vermieden werden, dass die Tiere als ihre eigene interne Kontrolle genutzt werden können.

Insgesamt haben in der biomedizinischen Bildgebung verschiedene Verfahren recht unterschiedliche Stellenwerte und ergänzen sich darüber hinaus auch teilweise, wie z. B. hochaufgelöste radiologische und hochsensitive nuklearmedizinische Verfahren. Da sich dieses Feld einem schnellen Wandel unterzieht, ist es für potenzielle Anwender, aber auch für klinische Radiologen und Nuklearmediziner, äußerst wichtig, zumindest ein grundlegendes Verständnis für das Feld zu haben, um so mögliche Anwendungen fundiert diskutieren zu können. Daher ist es das Ziel dieser Übersicht, einen Überblick über die radiologischen und nuklearmedizinischen Möglichkeiten zur Bildgebung an Tieren, mit speziellem Blick auf die Fusionierung der Informationen aus beiden Fächern, zu liefern.

\section{Radiologische Applikationen \\ $\nabla$}

Im Prinzip stehen für die tierexperimentelle bildgebende Forschung die gleichen Modalitäten zur Verfügung wie sie auch für die humane Anwendung existieren. Dies sind insbesondere die Computertomografie (CT) und die Magnetresonanztomografie (MRT) [1]. Im folgenden Abschnitt sollten beispielhaft einige Applikationen dieser Modalitäten sowie deren Vor- und Nachteile beschrieben werden.

\section{Magnetresonanztomografie}

Die frühe Entwicklung der MRT fand hauptsächlich in Kleintierexperimenten statt, da zu dieser Zeit nur Magnetresonanzspektrometer mit kleinen Bohrungen zur Verfügung standen und diese deshalb nur für Mäuse geeignet waren [2, 3]. Schon bald wurden humane und Kleintiertomografen parallel weiterentwickelt. Während dies in der Anfangszeit die wesentlichen Gründe für die Bildgebung vor allem an Mäusen waren, nutzt man heute auch aus, dass spezielle miniaturisierte Radiofrequenz(RF-)spulen für die tierexperimentelle Bildgebung genutzt werden können. Solche Spulen haben den Vorteil, dass sie durch ihre Nähe zum Objekt und dem geringen Totraum in ihrem Inneren eine höhere Sensitivität für die vom Untersuchungsobjekt ausgehenden magnetischen Felder und damit ein höheres Signal-zu-Rausch Verhältnis (signal to noise ratio SNR) haben [4].

Heutzutage werden sowohl dedizierte Kleintier- als auch klinische MR-Tomografen zur tierexperimentellen Bildgebung verwandt. Die dedizierten Tomografen haben die Vorteile, dass sie oft eine bessere Bildqualität liefern und eine größere Flexibilität für experimentelle Untersuchungen bieten. Nachteile bestehen in der eingeschränkten Nutzbarkeit nur für Tiere bis zu einer bestimmten Größe, der Notwendigkeit von speziell ausgebildeten Anwendern vorzugsweise mit naturwissenschaftlichem Hintergrund und der beschränkten Verfügbarkeit. Auf der andere Seite haben die klinischen Tomografen Vorteile bei der Umsetzung von tierexperimentellen Protokollen in humane Anwendungen, in der einfachen Standardisierung der Untersuchungen, der einfachen Bedienung der Geräte und der ubiquitären Verfügbarkeit in jedem universitären Klinikum. Nachteile bestehen in der geringeren Bildqualität, der Notwendigkeit für Spezialhardware, wie z.B.
RF-Spulen, und der geringen Sensitivität für extrinsische Kontrastmittel [4-9].

Insgesamt findet man heute ein sehr breites Anwendungspektrum für die tierexperimentelle MRT. Neben den am weitesten verbreiteten Anwendungen zur Bestimmung der Tumorgröße, auch im zeitlichen Verlauf, in verschiedenen Tiermodellen, gibt es zahlreiche Studien zur neurofunktionellen MRT, zu Genese und Behandlung von inflammatorischen Erkrankung, zu Lungenerkrankungen, wie Asthma oder Emphysem, und vielen anderen mehr [10-13].

\section{Computertomografie}

Im Gegensatz zur MRT gab es bei der Entwicklung der CT keine Vorläufergeräte, sodass von Anfang der Bau von Computer-Tomografen für humane Anwendungen im Vorgrund stand. In den letzten Jahren haben allerdings verschiedene Firmen damit begonnen, spezielle Kleintier-CTs zu entwickeln. Mit der heute zur Verfügung stehenden Technologie können isotrope Datensätze von Mäusen mit einer Auflösung von $100 \times 100 \times 100 \mu \mathrm{m}$ in wenigen Minuten aufgenommen werden. Es wurde bereits gezeigt, dass auch isotrope Auflösungen von $50 \mu \mathrm{m}$ möglich sind. Dabei erreicht die Strahlenexposition dann ein Niveau, welches schon als invasiv angesehen werden muss, und von vielen Tieren auch nicht mehr überlebt wird. Das größte Interesse lag bisher auf Untersuchungen des Skeletts und des Respiratorischen Systems [14-16].

Hohe räumliche und zeitliche Auflösung machen die CT zu einem idealen Werkzeug, um große Tierpopulationen auf morphologische, „phenotypische“ Veränderungen zu untersuchen. Dazu ist es allerdings noch notwendig Systeme zu entwickeln, welche solche „Screening“-Untersuchungen unterstützen und einen hohen Durchsatz an Tieren in kurzer Zeit ermöglichen. Dabei muss vor allem das Hantieren mit den anästhesierten Tieren automatisiert werden. Zusätzlich stellt sich dann natürlich auch die Frage nach automatischer Evaluationssoftware. Wenn man bedenkt, dass ein Datensatz einer Maus mit einer isotropen Voxelgröße von $50 \mu \mathrm{m}$ ca. $10^{9}$ Voxeln entspricht, benötigt man sehr effiziente Bildnachverarbeitungssoftware, um diese Datenmengen zu rekonstruieren. Darüber hinaus müssen in einem Screeningprozess eine große Anzahl von Tieren in kurzer Zeit auf bestimmte Merkmale hin untersucht werden. Dies kann effizient auch nur von automatischen Softwarelösungen geleistet werden [17]. Für die CT gilt, dass ihre größten Vorteile die hohe räumlich und zeitliche Auflösung sowie die relativ kostengünstigen und platzsparenden Kleintier-Computer-Tomografen sind. Ihre Nachteile bestehen in geringem Weichteilkontrast, hohen Dosen ionisierender Strahlung und geringer Sensitivität für extrinisische Kontrastmittel.

Im Allgemeinen lässt sich sagen, dass diese Methoden ihre Stärken in der morphologischen Darstellung kleinster Prozesse besitzen. Ihre hohe räumliche und Kontrastauflösung erlauben eine exzellente Darstellung von kleinsten, anatomische abgrenzbaren Prozessen. Allerdings liegt da auch ihre größte Schwäche. Sie sind für eine Kontrastmittelanreicherung wenig sensitiv und benötigen daher große Mengen Kontrastmittel, welches sich am Ort des zu beobachtenden Krankheitsprozesses stark anreichern muss. Dies führt oft dazu, dass kleine Prozesse nicht erkannt werden bzw. dass Veränderungen zwar erkannt, aber nicht spezifisch als maligne oder benigne eingeordnet werden können. 


\section{Nuklearmedizinischen Applikationen}

$\nabla$

\section{Single Photonen Emissions Computer Tomography (SPECT)}

In der Nuklearmedizin werden Photonenstrahlen zur Bildgebung benutzt. Die „Single Photonen Emissions Computer Tomography“ benutzt, die von $\gamma$-Strahlern emitierten Photonen. Dabei sind die Detektoren der $\gamma$-Kameras darauf ausgelegt, Energien im Bereich von 80-400 keV zu detektieren, wobei der ideale Energiebereich von 120-200 keV geht. Die Nuklide, die eingesetzt werden, sind in - Tab. 1 aufgelistet. Es gilt dabei zwei Arten von Nukliden zu unterscheiden. Die Radiometalle müssen in der Regel über einen Chelator in die gewünschte Verbindung zur Diagnose gebracht werden. Als Beispiele seien hier die ${ }^{99 \mathrm{~m}} \mathrm{Tc}$-Phosphonate zur Detektion von erhöhtem Knochenumsatz (z.B. Knochenmetastasen oder Osteomelytis) oder lipophile ${ }^{99 \mathrm{~m}} \mathrm{Tc}-$ Verbindungen zur Hirnperfusionsmessungen erwähnt. Die Metalle können auch über den Chelator an spezifische Moleküle gekoppelt werden. Die Radioiodnuklide können für die Detektion der Natriumiodidsymporters direkt eingesetzt werden. Daneben ist es auch möglich, das Iod über Tyrosin in Peptide und Proteine einzubauen. Doch sind diese Markierung in der Regel metabolisch unstabil und das Iod wird freigesetzt [18]. Neuere Ansätze zeigen, dass es auch möglich ist stabile Markierungen von Proteinen mit Iod zu erreichen [19].

Um nur die Photonen zu detektieren, die in einem Winkel von ca. $90^{\circ}$ auf den Detektor (in der Regel ein Thallium dotierter Natriumiodidkristall) treffen und damit eine direkte Abbildung vom Objekt zu erhalten, wird ein Parallellochkollimator vor den Detektor montiert ( $\bullet$ Abb. 1A). Mit den Parallellochkollimator wird ein Objekt ohne Vergrößerung auf den Detektor abgebildet. Je nach Energie des eingesetzten Nuklids werden verschiedene Kollimatoren mit breiteren Septen verwendet. Dadurch verringert sich die Auflösung mit höherer Energie. Bei Tc-99 m (140 keV) kann man in der klinischen Routine in Abhängigkeit der Aktivitätskonzentration im Zielgewebe von einer Auflösung von deutlich unter $1 \mathrm{~cm}$ ausgehen [20].

Diese Auflösung ist natürlich für die Bildgebung bei Kleintieren unzureichend. Eine Möglichkeit dies zu umgehen ist die Anwendung von Pinholekollimatoren, die nach dem gleichen Prinzip wie eine Kamera Obscura funktioniert und eine Vergrößerung des Abbildungsobjekts auf den Detektor ermöglicht $($ Abb. 1B). Je größer der Quotient zwischen dem Abstand Detektor-Kollimator und Detektor-Abbildungsobjekt ist, um so besser die Vergrößerung. Dadurch kann man Auflösungen im Bereich von unter einem Millimeter erreichen.

\begin{tabular}{|c|c|c|c|c|c|c|}
\hline Nuklid & Anwendung & $\begin{array}{l}\gamma \text {-Energie } \\
{[\mathrm{keV}]}\end{array}$ & andere Strahler & Halbwertszeit (h) & Eigenschaften & $\begin{array}{l}\text { Tab. } 1 \text { Radionuklide für die An- } \\
\text { wendung bei SPECT und PET }\end{array}$ \\
\hline Tc-99 m & SPECT & 141 & - & 6 & Metall & \\
\hline Ga-67 & SPECT & $\begin{array}{c}93.3 \\
185 \\
300\end{array}$ & - & 79,2 & Metall & \\
\hline $\ln -111$ & SPECT & $\begin{array}{l}171 \\
245\end{array}$ & - & 67,2 & Metall & \\
\hline $\mid-123$ & SPECT & 159 & - & 13,2 & Halogen & \\
\hline $\mid-125$ & in vitro/(SPECT) & $\begin{array}{l}27 \\
31\end{array}$ & - & 1425,6 & Halogen & \\
\hline $\mid-131$ & SPECT & 364 & $\beta^{-}($Emax: $660 \mathrm{keV})$ & 192 & Halogen & \\
\hline$C-11$ & PET & 511 & $\beta^{+}($Emax: $960 \mathrm{keV})$ & 0,34 & organisch & \\
\hline $0-15$ & PET & 511 & $\beta^{+}($Emax: $1740 \mathrm{keV})$ & 0,034 & organisch & \\
\hline$F-18$ & PET & 511 & $\beta^{+}($Emax: $630 \mathrm{keV})$ & 1,8 & Halogen & \\
\hline Ga-68 & PET & 511 & $\beta^{+}($Emax: $1899 \mathrm{keV})$ & 1,13 & Metall & \\
\hline I-124 & PET & 511 & $\beta^{+}($Emax: $1534 ; 2137 \mathrm{keV})$ & 100,2 & Halogen & \\
\hline
\end{tabular}
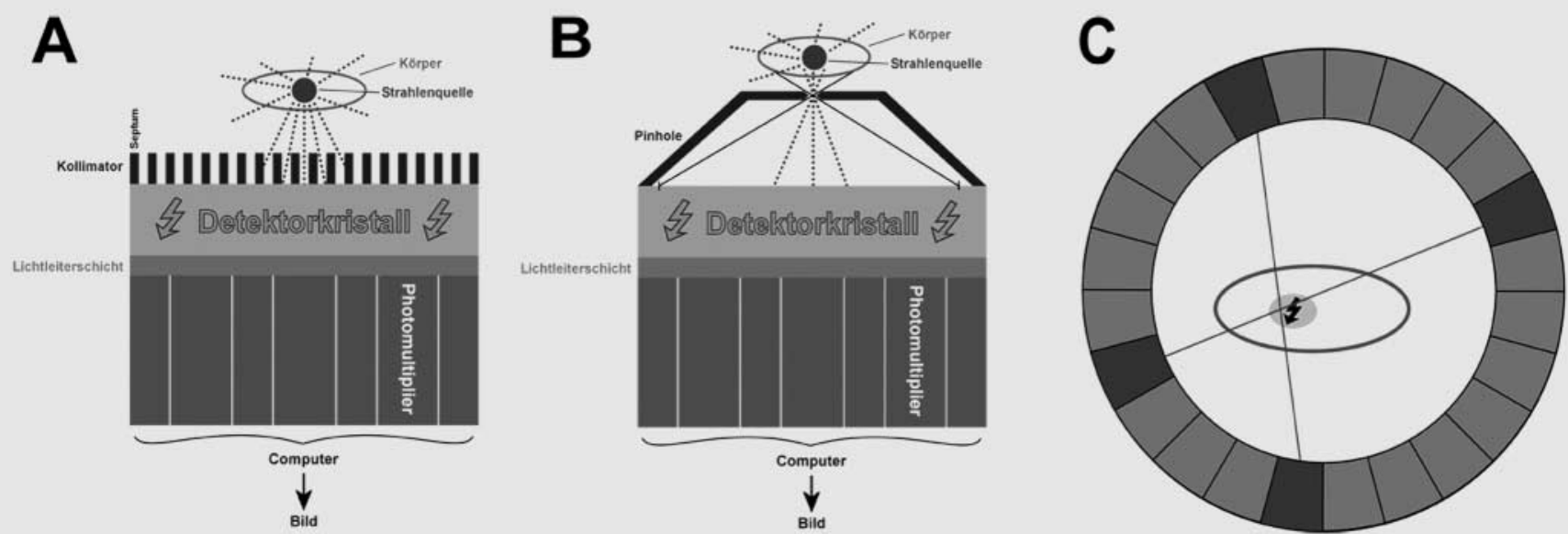

Abb. 1 A Detektorkopf einer $\gamma$-Kamera mit einem Paralellochkollimator. B Detektorkopf einer $\gamma$-Kamera mit einem Pinhole-Kollimator. C Detektorring einer PET-Kamera. 
Diese höhere Auflösung bezahlt man allerdings durch einen Verlust an Sensitivität. Diesen Verlust kann durch den Einsatz von Multipinhole-Kollimatoren entgegengewirkt werden. Es können Kollimatoren mit bis zu 10 Pinholes verwendet werden [20].

Die dreidimensionale Information erhält man dadurch, dass die Detektoren um das Abbildungsobjekt rotieren. Durch eine iterative Rekonstruktion ist es möglich, ein dreidimensionales Abbild zu erhalten.

\section{Positronen Emissions Tomography (PET)}

Eine weitere nuklearmedizinische Anwendung ist die Positronen Emissions Tomography (PET). Dabei wird von den Nukliden eine Positron $\left(\beta^{+}\right)$eine Antiteilchen $\mathrm{zu}$ einem Elektron emittiert, welches dann nach einer von der energieabhängigen Wegstrecke mit einem Elektron annihiliert. Das heißt, die Masse des Elektrons und des Positron werden nach der Formel von Einstein $E=m c^{2}$ in Energie in Form von Photonen umgewandelt und zwar werden zwei Photonen oder $\gamma$-Strahlung in einem Winkel von $180^{\circ}$ mit einer Energie von jeweils $511 \mathrm{keV}$ ausgesendet. Die Detektion erfolgt durch zwei gegenüberliegende Detektoren in einem Ring von Detektoren ( Abb. 1C). Dadurch ist auch eine Ortsbestimmung möglich. Moderne Geräte benutzen die Zeitdifferenz zwischen dem Ansprechen der gegenüberliegenden Detektoren zur genauen Ortsbestimmung („time of flight“ TOF). Als Nuklide kommen die in der $\bullet$ Tab. 1 aufgeführten in Frage. ${ }^{11} \mathrm{C}$ hat den Vorteil, dass es in jedes organische Molekül eingebaut werden kann, ohne dessen chemischen und damit biologischen Eigenschaften zu verändern. Der Nachteil ist natürlich die kurze Halbwertszeit von 20 min. Dies bedingt, dass dieses Nuklid wie auch ${ }^{15} \mathrm{O}$ nur eingesetzt werden kann, wenn ein Zyklotron zur Produktion vor Ort ist. Dies gilt eingeschränkt ebenfalls für ${ }^{18} \mathrm{~F}$ mit knapp $2 \mathrm{~h}$ Halbwertszeit. ${ }^{18} \mathrm{~F}$-Deoxy-Glukose ist im Moment das meist gebrauchte PET-Radiopharmaka, da damit der Glukose Metabolismus in verschiedenen Geweben untersucht werden kann. ${ }^{68} \mathrm{Ga}$ ist, wie das ${ }^{99 \mathrm{~m} T c}$, ein Nuklid, das durch einen Generator gewonnen werden kann und somit ständig zur Verfügung steht. Doch ist im Moment die Anwendung noch begrenzt, da die geeigneten Vorläufersubstanzen noch nicht zur Verfügung stehen. Die Auflösung für PET liegt im Bereich von $4 \mathrm{~mm}$ für klinische Geräte und $1 \mathrm{~mm}$ für Kleintierbildgebung [21].

\section{Bildfusionen}

\section{$\nabla$}

Die beschriebenen Methoden haben sowohl Vor- als auch Nachteile. Die nuklearmedizinischen Methoden haben eine sehr hohe Sensitivität (SPECT: $10^{-10}-10^{-11} \mathrm{M}$; PET: $10^{-11}-10^{-12} \mathrm{M}$ ) und da-

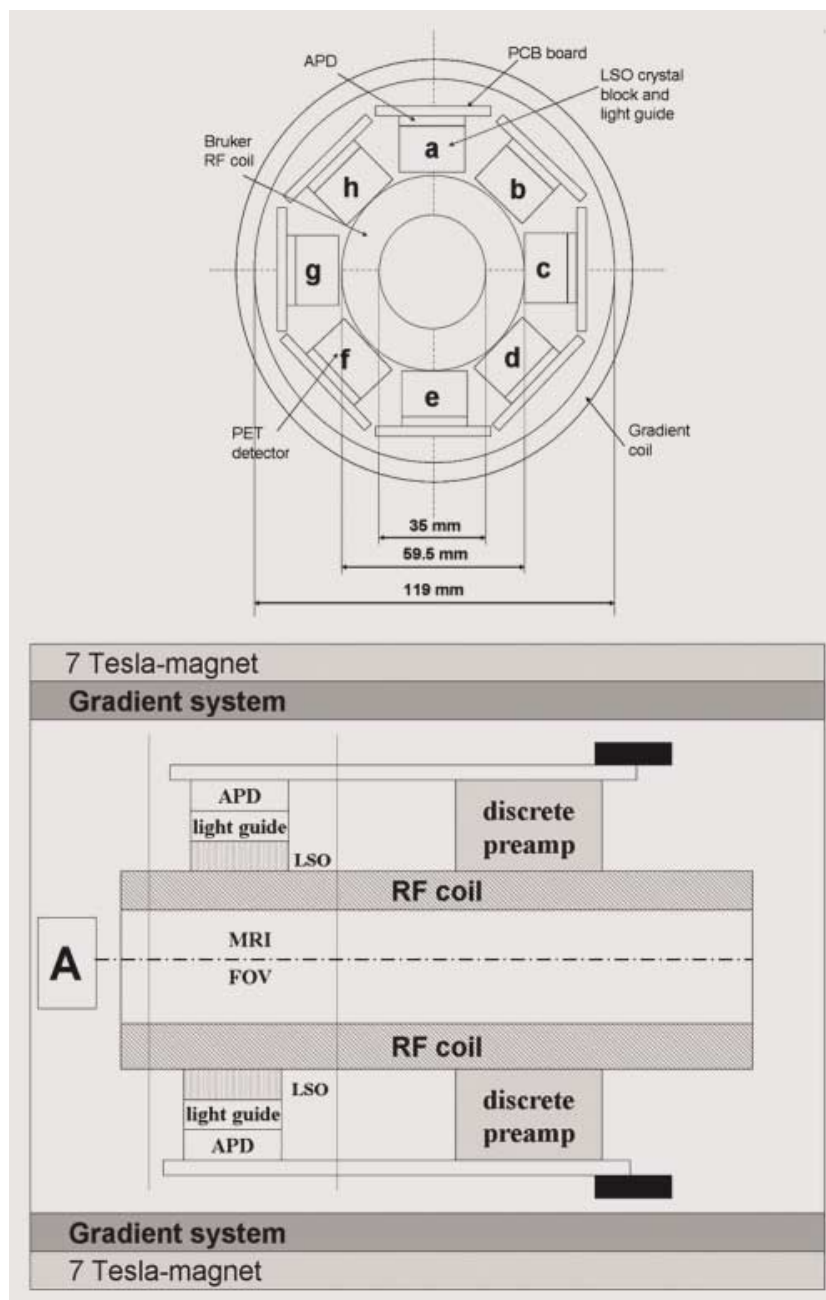

Abb. 2 Die Zeichnung zeigt den PET-Ring besteht aus kompakten APD Detektoren, welcher in die Bohrung des MRTs eingeführt werden kann. $\mathrm{PCB}=$ Printed Circuit Board. Reprint erlaubt mit freundlicher Genehmigung von der "Society of Nuclear Medicine“ aus [24].

Tab. 2 Eigenschaften kombinierter nuklearmedizinischer und radiologischer Verfahren

\begin{tabular}{|c|c|c|c|c|c|}
\hline Modalität & $\begin{array}{l}\text { Anzahl von } \\
\text { räumlich getrennten } \\
\text { MR-Tomografen }\end{array}$ & Datenakquisition & Koregistrierung & Vorteile & Nachteile \\
\hline $\mathrm{PET} / \mathrm{CT}$ & 1 & sequenziell & Tischposition & $\begin{array}{l}\text { CT-Daten können zur } \\
\text { Schwächungskorrektur } \\
\text { genutzt werden }\end{array}$ & $\begin{array}{l}\text { Kombination zu einem } \\
\text { MR-Tomografen verlängert } \\
\text { die Gantry }\end{array}$ \\
\hline SPECT/CT & 1 & sequenziell & Tischposition & & \\
\hline SPECT/MR & 2 & sequenziell & Marker/Anatomie & & $\begin{array}{l}\text { hoher Zeitaufwand bei } \\
\text { räumlich getrennten } \\
\text { MR-Tomografen }\end{array}$ \\
\hline PET/MR & 1 & simultan & $\begin{array}{l}\text { keine Registrierung } \\
\text { notwendig, da simul- } \\
\text { tane Akquisition }\end{array}$ & $\begin{array}{l}\text { durch simultane Daten- } \\
\text { akquisition: } \\
\text { - Geschwindigkeit } \\
\text { - automatische Regis- } \\
\text { trierung }\end{array}$ & $\begin{array}{l}\text { Durchmesser der Gantry } \\
\text { wird durch PET-Insert } \\
\text { wesentlich verringert } \\
\text { MR-Daten können nicht zur } \\
\text { Schwächungskorrektur } \\
\text { genutzt werden }\end{array}$ \\
\hline
\end{tabular}




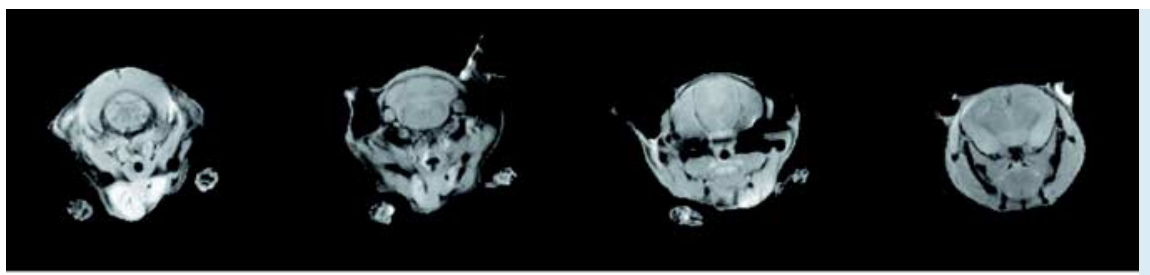

Abb. 3 zeigt PET- und MRT-Bilder, welche simultan in einem 7T-MR-Tomografen mit einem PETInsert aufgenommen wurden. Obere Reihe: MRTBilder; Mittlere Reihe: PET-Bilder; Untere Reihe: Fusionierte PET/MRT-Bilder. Reprint erlaubt mit freundlicher Genehmigung von der „Society of Nuclear Medicine" aus [25].
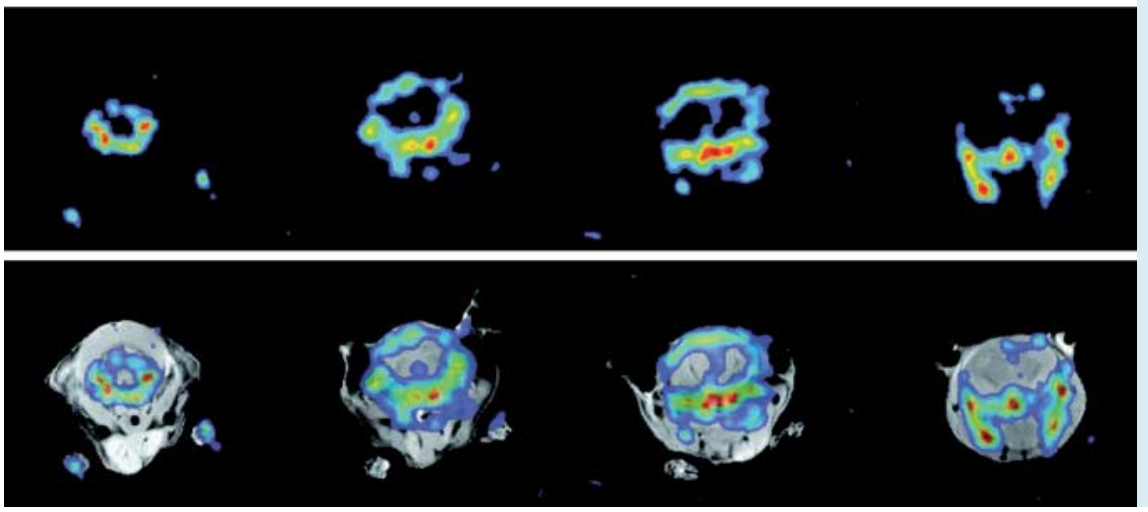

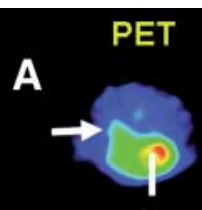

h

B

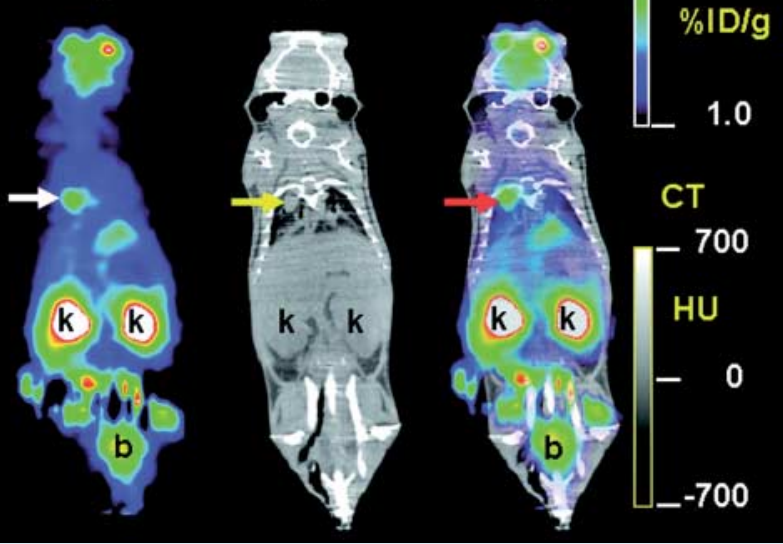

Abb. 4 Transverse (A) und coronale (B) Schnitte eines Lungenmetastasenmodells eines Melanoms. Die PET-Studie wurde mittels ${ }^{18} \mathrm{~F}$-Deoxyglukose durchgeführt. Eine Lungenmetastase wird durch einen weißen (PET), gelben (CT) und roten (PET/CT) Pfeil bezeichnet. Die physiologische FDGAnreicherung in Herz (h), Nieren (k) und Blase (b) ist ebenfalls zu sehen. Reprint erlaubt mit freundlicher Genehmigung von der „Society of Nuclear Medicine“ aus [28].

mit die Möglichkeit, funktionelle Bildgebung zu machen. Allerdings ist die genaue Lokalisation einer erhöhten Anreicherung von Radiotracern schwierig und die Auflösung liegt nur im mmBereich. Die radiologischen Methoden bieten dagegen den Vorteil von genauen morphologischen Informationen mit einer hohen Auflösung, während hier die Sensitivität geringer ist (z.B. MRT: $\left.10^{-3}-10^{-5} \mathrm{M}\right)$ und damit eine funktionelle molekulare Bildgebung schwieriger ist [22]. Den idealen Informationsgehalt erhält man also, wenn man die radiologische und nuklearmedizinische Bildgebung fusioniert.
Solche Fusionen werden in der klinischen Anwendung schon länger durchgeführt. Die Bilder der verschiedenen Modalitäten wurden in den Anfängen auf dem Computer überlagert. Die Firmen entwickelten in der Folge kombinierte Geräte wie das PET/CT. In der Folge wurde auch ein SPECT/CT und jetzt als neuste Generation kommt ein PET/MRT auf den Markt.

Alle diese Modalitäten haben vor allem das Ziel, die hohe räumliche Auflösung der CT und MRT mit der hohen Sensitivität der SPECT und PET zu kombinieren. Dies bringt neben diesem herausragenden Vorzug noch weitere mit sich. Allerdings erkauft man sich immer auch Nachteile ( Tab. 2). So erhält man in einem PET/CT aus den CT-Datensätzen nicht nur morphologische Informationen, sondern gleichzeitig auch räumlich hochaufgelöste Dichteinformationen, welche zur Schwächungskorrektur des PET's genutzt werden können. Die SPECT/MRT bringt zusätzlich zur hohen räumlichen Auflösung des MRT auch noch seine hohe Kontrastauflösung und die Möglichkeit, funktionelle, quantitative Daten zu erheben. Da sich die beiden Modalitäten aber gegenseitig beeinflussen würden, müssen räumlich voneinander getrennt aufgestellt werden. Dadurch wird der Aufwand zur Registrierung der Bilder größer und die Untersuchungszeit länger, da die Untersuchungsobjekte vom PET zum MR-Tomograf transportiert werden müssen. Die PET/MR beschleunigt die Datenakquisition erheblich, da in dieser Modalität beide Datensätze simultan aufgenommen werden können. Dies macht eine nachträgliche Koregistrierung ebenfalls unnötig. Auf der anderen Seite wird das Nutzvolumen der MR-Gantry kleiner, da das der PET-Ring als Insert in diese eingeschoben wird. Des Weiteren können die MR-Daten noch nicht zur Schwächungskorrektur der PET-Daten genutzt werden. Hierzu gibt es allerdings bereits Lösungsansätze.

Die meisten der kombinierten Modalitäten führen zu einer Platzersparnis, da anstatt zweier MR-Tomografen nun nur noch einer benötigt wird. Einzige Ausnahme ist SPECT/MR, da sich hierbei die beiden MR-Tomografen gegenseitig bei der Datenakquisition beeinflussen. Die Zusammenführung zweier Geräte hat aber gleichzeitig den Nachteil, dass sie nicht mehr getrennt genutzt werden können. D.h. ein PET/CT kann zwar einerseits als PET und anderer- 


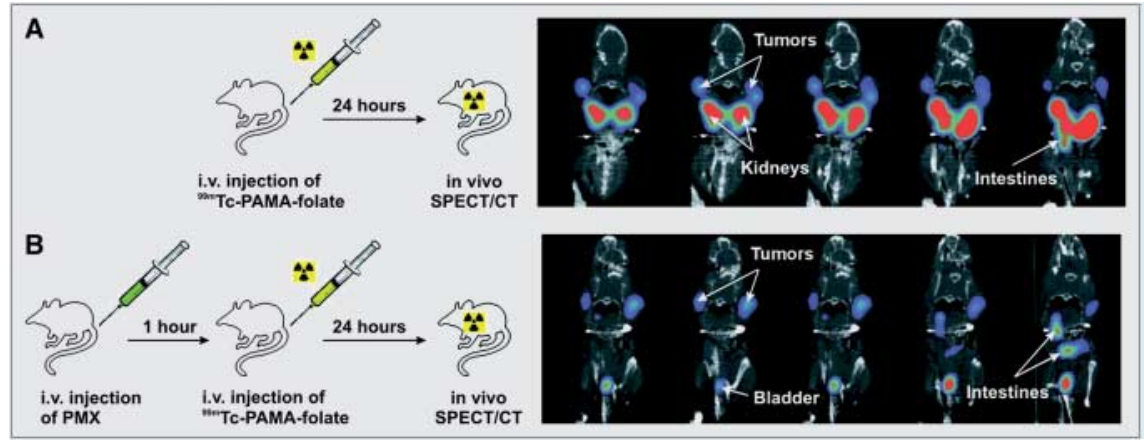

Abb. 5 Die Überlagerung von SPECT-Bildern mit $99 \mathrm{~m}$ Tc-Folat und CT zeigt, dass sich das Verhalten des Radiopharmakons in der unteren Reihe nach der Preinjektion von Pemetrexed in den Nieren deutlich ändert, während der Uptake im Tumor ähnlich bleibt. Reprint erlaubt mit freundlicher Genehmigung von der "Society of Nuclear Medicine“ aus [29].

seits als CT betrieben werden, aber nicht simultan an zwei verschiedenen Untersuchungsobjekten.

\section{Beispiele}

PET/MRT

Die Kombination von PET- und MR-Bildgebung hat wie vorangegangen beschrieben viele Vorzüge, da die beiden Modalitäten komplementäre Informationen liefern [23]. PET ist durch die Verbindung von positronenemittierenden Radionukliden mit biologisch relevanten Molekülen extrem sensitiv für eine Vielzahl von biologischen Prozessen. Die MRT auf der anderen Seite liefert eine hohe Ortsauflösung, Information über physiologische Parameter, spektroskopische Daten über metabolische und biochemische Prozesse. Auf der anderen Seite ist es im Vergleich zur PET/CT weitaus schwieriger, die beiden Modalitäten in eine zu integrieren, da sie sich durch ihre Eigenschaften gegenseitig beeinflussen. So stört das magnetische Feld die PET-Akquisition (Photomultiplier arbeiten nicht) und die in das Magnetfeld eingebrachten Sensoren stören die Homogenität des Feldes.

Zur Zeit gibt es zwei Ansätze zur Realisierung einer PET/ MRT-Modalität: 1 . Anstatt die Photomultiplier direkt im MRT anzubringen werden nur die Szintillationskristalle in das Magnetfeld eingebracht und deren Signal über Lichtleiter zu Photomultipliern außerhalb des Feldes geleitet und dort verstärkt. Damit fällt die gegenseitige Beeinflussung weg. Allerdings geht damit auch ein hoher Signalverlust von bis $75 \%$ in den Lichtleitern einher [9, 11]. 2. Anstatt Photomultiplier zu verwenden, werden Lawinenphotodioden (Avalanche Photo Diode $=$ APD) eingesetzt. Diese werden vom Magnetfeld nicht mehr beeinflusst und sind vor allem auch wesentlich kleiner als Photomultiplier. Damit gelang es nun einen Ring als PET-Insert für das MRT aufzubauen ( Abb.2) [24]. Die simultane Bildgebung mittels dieser PET/MRT-Kombination wurde dann in einem Mausmodell als proof of principle gezeigt ( $\bullet$ Abb. 3) [25].

\section{PET/CT}

Bei Kombination von PET/CT in einem Gerät sind die geringsten technischen Schwierigkeiten zu überwinden und ist aus diesem Grund das gebräuchlichste Kombinationsgerät sowohl in der Klinik wie auch in der Kleintierbildgebung [26, 27]. Dementsprechend viele verschiedene Studien sind vorhanden. Das Paper aus der Gruppe von Gambhir [28] zeigt die Möglichkeiten des kombinierten Geräts auf. Die Bilder zeigen die Detektion einer Lungenmetastase in einem Melanommodell. Nach der Injektion in die Schwanzvene von Melanomzellen in einer SCID-Maus können die Metastasen nach 45 Tagen detektiert werden. Dabei zeigt das PET-Bild mit ${ }^{18}$ F-Fluorodeoxyglukose (FDG) den Hypermetabolis- a

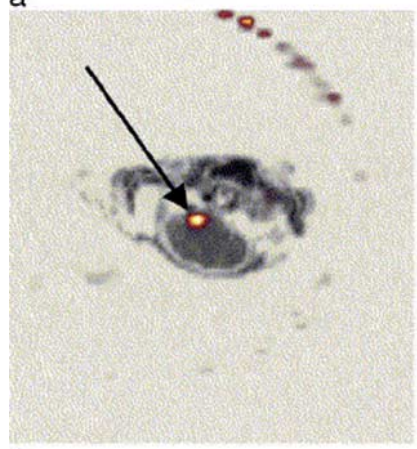

C

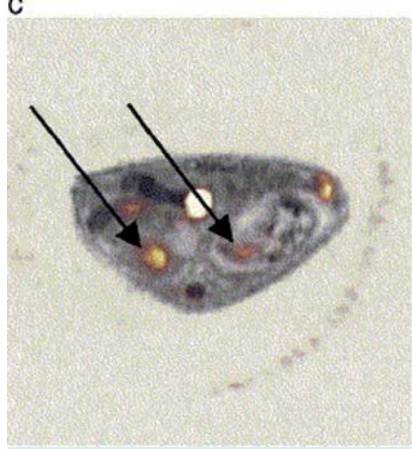

b

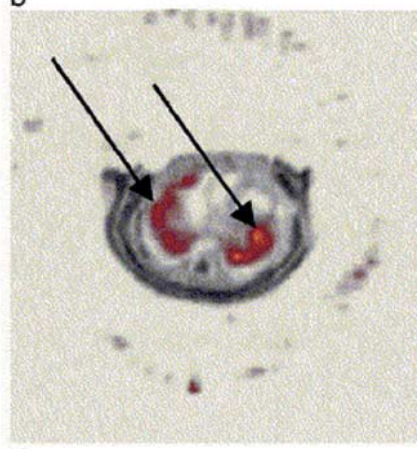

d

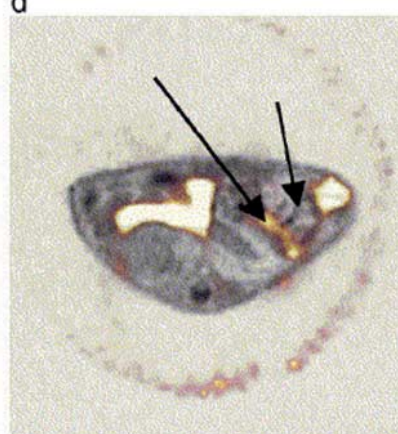

Abb. 6 Eine Fusion von Bildern aus SPECT und MRT. Das SPECT-Bild zeigt die Bioverteilung von $4 \mathrm{~h}$ p. i. von $35 \mathrm{MBq}{ }^{111}$ In-DTPA-Lys ${ }^{40}$-Exendin 4. Durch die MRT-Bilder konnten der Uptake den Geweben zugeordnet werden: Hypophyse (a, Pfeil), Lunge (b, Pfeile), Nebennieren (c, Pfeile), und Pankreas (d, Pfeil, für die bessere Orientierung wurde die Milz mit einem kleinen Pfeil gekennzeichnet). Reprint von [30] mit Erlaubnis von Elsevier.

mus in der Metastase, während das CT die genaue Lokalisation ermöglicht ( Abb.4). Dabei wird neben einer genauen Lokalisation des Tumors durch das CT auch die zusätzliche Information gewonnen, dass der Tumor einen hohen metabolischen Umsatz aufweist und dadurch eine hohe Maglinität erreicht.

\section{SPECT/CT}

In der Folge von PET/CT Geräten wurden auch SPECT/CT Geräte entwickelt [26]. So sind verschieden kommerzielle Geräte zur Kleintierbildgebung erhältlich. Die Gruppe von Schibli hat SPECT/CT benutzt, um den Einfluss von Methotrexate (MTX, Antifolate) auf die Bioverteilung eine ${ }^{99 \mathrm{~m}} \mathrm{Tc}$ markierten Folateanalogon ( ${ }^{99} \mathrm{mTc}$ TAMA-folate) zu untersuchen [29]. Dabei wurde festgestellt, dass der Uptake im Tumor entgegen den Erwartungen leicht niedriger war, der hohe Uptake in den Nieren jedoch dramatisch reduziert wurde. Dies führt zur Schlussfolgerung, dass durch die Vorbehandlung mit MTX auch eine Therapie ohne Nierentoxizität möglich ist ( $\bullet$ Abb.5). Diese Studie zeigt 

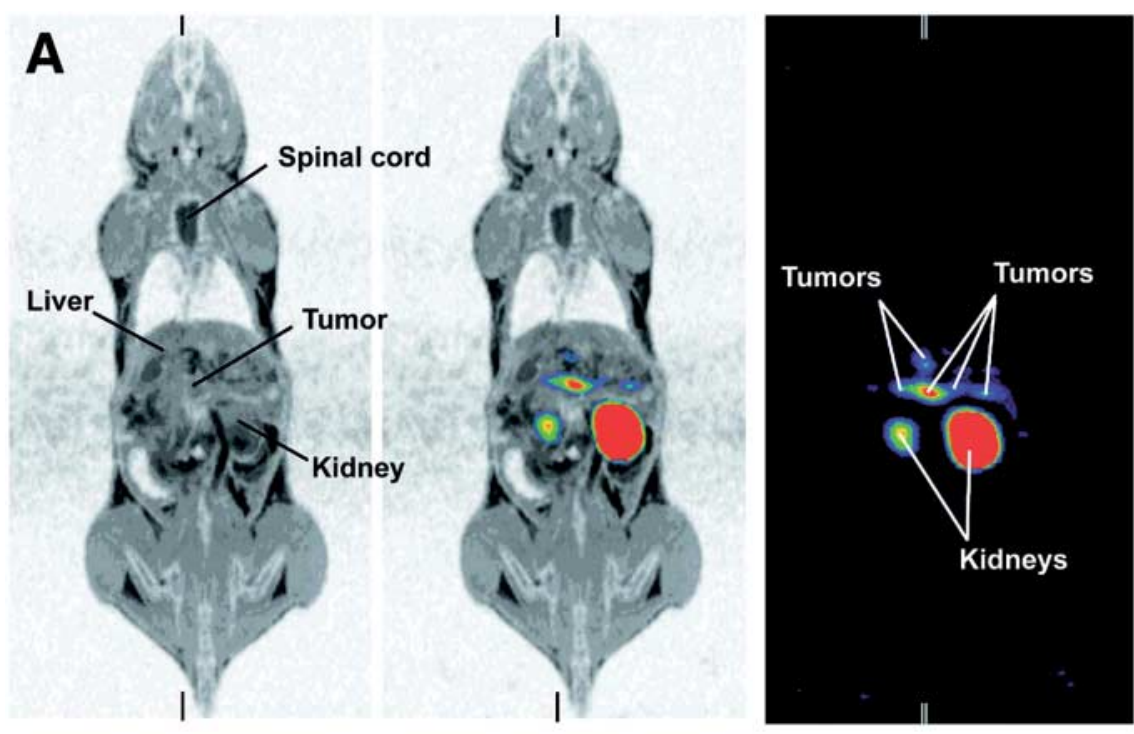

Abb. 7 Detektion von Insulinomen (d: 3,2 mm) durch ${ }^{111}$ In-DTPA-Lys ${ }^{40}$-Exendin 4 im Rip1Tag2 Mausmodell $4 \mathrm{~h}$ nach Injektion Reprint erlaubt mit freundlicher Genehmigung von der „Society of Nuclear Medicine" aus [31].

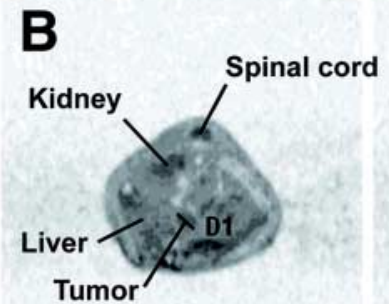

D1: distance $=3.2 \mathrm{~mm}$

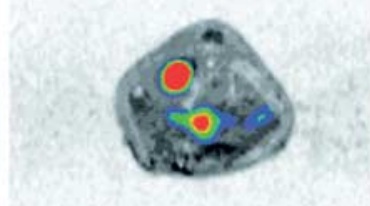

sehr schön die Anwendung der kombinierten Methode bei der Entwicklung von Methoden zur Beeinflussung der Bioverteilung. Durch diese nichtinvasiven Methoden ist es möglich, die Anzahl der benötigten Tiere dramatisch zu reduzieren. Durch die kombinierte Methode ist es auch möglich, die genaue Lokalisation des hohen Uptakes zu ermitteln. Die Aufnahmen mit dieser hohen Auflösung im SPECT ist nur möglich durch die Anwendung eines Pinhole-Kollimators. Die Kombination der radiologischen und nuklearmedizinischen Methoden macht auch nur Sinn, wenn die Auflösung für die nuklearmedizinischen Methoden im niedrigen mm-Bereich liegen.

\section{SPECT/MRT}

Hier treten dieselben Schwierigkeiten auf wie beim PET/MRT. Aus diesem Grund ist die Entwicklung dieser Geräte für Kleintiere noch im Forschungsstadium. Dies bedingt, dass die Tiere in einer fixierten Position transportiert werden müssen und danach die Bilder anhand von Markern oder anatomischen Merkmalen fusioniert werden müssen. Die Abbildung zeigt die Bioverteilung ${ }^{111}$ In-DTPA-Lys40-Exendin 4 in einer normalen Maus. Exendin-4 ist ein Agonist vom „Glucagon-Like-Peptide 1“ (GLP-1). In Nagern werden die Rezeptoren dieses Peptids in verschiedenen Geweben exprimiert. Neben dem Uptake im Darm, kann man auch spezifischen Uptake in der Hypophyse, Lunge, Nebenniere und im Pankreas sehen. Die Zuordnung der Verteilung des radioaktiven Peptids konnte nur aufgrund der fusionierten Bilder entstehen ( Abb. 6) [30].

Die Effektivität der Kombination von SPECT und CT konnte auch im Rip1Tag2 Mausmodell gezeigt werden. Die Mäuse entwickeln ein spontanes Insulinom mit einer hohen Expressionsrate von GLP-1 Rezeptoren. Durch die Injektion von ${ }^{111}$ In-DTPALys40-Exendin 4 können selbst Metastasen im mm-Bereich nachgewiesen werden ( $\bullet$ Abb. 7) [31]. Durch die Kombination der beiden Modalitäten ist es hier möglich, die Größe der Tumore in vivo zu bestimmen und mit dem radioaktiven Uptake zu korrelieren.

\section{Schlussfolgerung}

$\nabla$

Die Kombination der radiologischen Methoden bringt einen erheblichen Gewinn an Informationen. Dies wird dazu führen, dass die Fusionierung von Bildgebungsverfahren weiter entwickelt wird, und es wird in viel stärkerem Maß zukünftig vor allem für die Kleintierbildgebung auch optische Bildgebungsmethoden beinhalten [32].

\section{Danksagung \\ $\nabla$}

Die Autoren möchten sich bei Herr Matthias Heck und Michael Lill für die Unterstützung bei der Produktion der $\bullet$ Abb. 1 bedanken.

\section{Widmung}

$\nabla$

Prof. Klaus Jochen Klose zum 60. Geburtstag gewidmet.

\section{Literatur}

1 Gluer CC, Barkmann R, Hahn HK et al. Parametric biomedical imagingwhat defines the quality of quantitative radiological approaches? Fortschr Röntgenstr 2006; 178: 1187-1201 
2 Lauterbur PC. All science is interdisciplinary - from magnetic moments to molecules to men (Nobel lecture). Angew Chem Int Ed Engl 2005; 44: 1004-1011

3 Lauterbur $P$. Image formation of induced local interactions: examples employing NMR. Nature 1973; 242: 190-191

4 Alfke $H$, Kohle S, Maurer $E$ et al. Analysis of mice tumor models using dynamic MRI data and a dedicated software platform. Fortschr Röntgenstr 2004; 176: 1226-1231

5 Dubowitz DJ, Tyszka JM, Sewry CA et al. High resolution magnetic resonance imaging of the brain in the dy/dy mouse with merosin-deficient congenital muscular dystrophy. Neuromuscul Disord 2000; 10: 292-298

6 Fayad ZA, Fallon JT, Shinnar $M$ et al. Noninvasive In vivo high-resolution magnetic resonance imaging of atherosclerotic lesions in genetically engineered mice. Circulation 1998; 98: 1541-1547

7 Jacobs RE, Ahrens ET, Dickinson ME et al. Towards a microMRI atlas of mouse development. Comput Med Imaging Graph 1999; 23: 15-24

8 Maxwell RJ, Nielsen FU, Breidahl T et al. Effects of combretastatin on murine tumours monitored by 31P MRS, $1 \mathrm{H}$ MRS and $1 \mathrm{H}$ MRI. Int J Radiat Oncol Biol Phys 1998; 42: 891-894

9 Slawson SE, Roman BB, Williams DS et al. Cardiac MRI of the normal and hypertrophied mouse heart. Magn Reson Med 1998; 39: 980-987

10 Heverhagen JT, Hahn HK, Wegmann $M$ et al. Volumetric analysis of mice lungs in a clinical magnetic resonance imaging scanner. MAGMA 2004; 17: 80-85

11 Simon G, drup-Link H, von Vopelius-Feldt J et al. MRT der Arthritis mit dem USPIO SH U 555 C: Optimierung des T1-Enhancements. Fortschr Röntgenstr 2006; 178: 200-206

12 Hess A, Sergejeva M, Budinsky L et al. Imaging of hyperalgesia in rats by functional MRI. Eur J Pain 2007; 11: 109-119

13 Bohm I, Traber F, Block W et al. Molekulare Bildgebung von Apoptose und Nekrose-Zellbiologische Grundlagen und Einsatz in der Onkologie. Fortschr Röntgenstr 2006; 178: 263-271

14 Graichen $H$, Lochmuller EM, Wolf $E$ et al. A non-destructive technique for 3-D microstructural phenotypic characterisation of bones in genetically altered mice: preliminary data in growth hormone transgenic animals and normal controls. Anat Embryol 1999; 199: 239-248

15 Kennel SJ, Davis IA, Branning J et al. High resolution computed tomography and MRI for monitoring lung tumor growth in mice undergoing radioimmunotherapy: correlation with histology. Med Phys 2000; 27: 1101-1107

16 Paulus MJ, Gleason SS, Kennel SJ et al. High resolution X-ray computed tomography: an emerging tool for small animal cancer research. Neoplasia $2000 ; 2: 62-70$
17 Balaban RS, Hampshire VA. Challenges in small animal noninvasive imaging. ILAR J 2001; 42: 248-262

18 Behr TM, Gotthardt M, Becker W et al. Radioiodination of monoclonal antibodies, proteins and peptides for diagnosis and therapy. A review of standardized, reliable and safe procedures for clinical grade levels $\mathrm{kBq}$ to $\mathrm{GBq}$ in the Gottingen/Marburg experience. Nuklearmedizin 2002; 41: 71-79

19 Stein R, Govindan SV, Mattes MJ et al. Improved iodine radiolabels for monoclonal antibody therapy. Cancer Res 2003; 63: 111-118

20 Beekman F, van der Have F. The pinhole: gateway to ultra-high-resolution three-dimensional radionuclide imaging. Eur J Nucl Med Mol Imaging 2007; 34: 151-161

21 Cherry SR. The 2006 Henry N. Wagner Lecture: Of mice and men (and positrons) - advances in PET imaging technology. J Nucl Med 2006; 47: $1735-1745$

22 Massoud TF, Gambhir SS. Molecular imaging in living subjects: seeing fundamental biological processes in a new light. Genes Dev 2003; 17: 545-580

23 Jacobs RE, Cherry SR. Complementary emerging techniques: high-resolution PET and MRI. Curr Opin Neurobiol 2001; 11: 621-629

24 Pichler BJ, Judenhofer MS, Catana C et al. Performance test of an LSOAPD detector in a 7-T MRI scanner for simultaneous PET/MRI. J Nucl Med 2006; 47: 639-647

25 Catana $C, W u$ Y, Judenhofer MS et al. Simultaneous acquisition of multislice PET and MR images: initial results with a MR-compatible PET scanner. J Nucl Med 2006; 47: 1968-1976

26 Del Guerra A, Belcari N. Advances in animal PET scanners. QJ Nucl Med 2002; 46: 35-47

27 Beyer T, Townsend DW, Brun T et al. A combined PET/CT scanner for clinical oncology. J Nucl Med 2000; 41: 1369-1379

28 Deroose CM, DE A, Loening AM et al. Multimodality Imaging of Tumor Xenografts and Metastases in Mice with Combined Small-Animal PET, Small-Animal CT, and Bioluminescence Imaging. J Nucl Med 2007; 48: 295-303

29 Muller C, Bruhlmeier M, Schubiger PA et al. Effects of antifolate drugs on the cellular uptake of radiofolates in vitro and in vivo. J Nucl Med 2006; 47: 2057-2064

30 Gotthardt $M$, Lalyko G, van Eerd-Vismale J et al. A new technique for in vivo imaging of specific GLP-1 binding sites: First results in small rodents. Regul Pept 2006; 137: 162-167

31 Wild D, Behe M, Wicki A et al. Lys40(Ahx-DTPA-111In)NH2 ] exendin-4, a very promising ligand for glucagon-like peptide- 1 (GLP-1) receptor targeting. J Nucl Med 2006; 47: 2025-2033

32 Grimm J, Wunder A. Molekulare Bildgebung: Stand der Forschung. Fortschr Röntgenstr 2005; 177: 326-337 